\title{
Exploring the Nature of Business Strategy Change for Mental Health Practices in the Age of COVID-19
}

\author{
De Andra Judge, The Chicago School of Professional Psychology, USA \\ (iD) https://orcid.org/0000-0001-8042-9365 \\ Darrell Norman Burrell, The Florida Institute of Technology, USA \& Capitol Technology University, USA \\ iD https://orcid.org/0000-0002-4675-9544
}

\begin{abstract}
The Convergence Lab (TCL) is a mental health medical group practice that provides group and individual therapy for its adult clients. TCL is a 5-year-old organization with five clinicians and one full-time office manager. As a result of COVID-19, TCL has experienced a 75\% decrease in revenue since patients are unable to complete their appointments in person. The paper examines how one mental health medical group practice used a management consultant to help the practice change its business strategy to respond to COVID-induced business challenges using automation and technology driven strategic approaches. This inquiry uses organizational development action research intervention and a content analysis of the literature to create real-world solutions that will have value to scholar practitioners that are doing applied research to help similar organizations that are challenged to respond to the global pandemic.
\end{abstract}

\section{KEYWORDS}

Business Strategy, Counseling, Management Consulting, Strategic Change, Strategic Planning, Tele-Counseling

\section{INTRODUCTION}

This case study examines real life data for a one mental health medical group that is has been given the fictious name of TCL at the request of the organization to protect its privacy and intellectual property. This case represents an intervention from a management consultant to help the practice respond to COVID-induced business challenges. Implementing a strategic market management process allows organizations to manage volatility occurring in an organization's external environment (Aaker, 2001).

Before COVID-19, TCL lacked a formalized strategy to address the pandemic. As a result, a management consultant brought the help given over $75 \%$ decline in revenue due to COVID-19. Unlike a single strategic planning event that is often completed once, annually, using a strategic change process allows organizations to continuously take in information, thereby deconstructing the organization from 
a predetermined, time-bound planning cycle (Aaker, 2001). The Convergence Lab used a management consultant to help the organizations adopt a strategy to respond better to COVID-19.

Strategic planning ideologies emerged and dominated the strategic management process between the 1960s and the 1980s (Emerald, 2003). The process encompasses a business's structured review of its customers and markets to craft a strategy to respond to changing market forces (Aaker, 2001). These organizational strategy changes might include plans to move into new markets or prioritize learning priorities and more (Aaker, 2001).

Strategic planning consultants have a myriad of tools for facilitating the planning process. One of these tools is a SWOT analysis, a review of an organization's Strengths, Weaknesses, Opportunities, and Treats to craft organizational strategies around opportunities, strengths, and competitive advantages. (Krogerus et al., 2018). SWOT analysis helped clarify organization members and design strategies to bolster strengths and mitigate weaknesses (Krogerus et al., 2018).

\section{Overview of the Problem}

TCL is a mental health medical group practice that provides group and individual therapy for its adult clients. TCL is a 5-year old organization with five (5) clinicians inclusive of one (1) full-time office manager, and five clinicians are full-time staff. As a result of COVID, TCL has experienced a 75\% decrease in revenue since patients cannot complete their appointments in person.

\section{Research Method}

This inquiry uses organizational development action research intervention and a content analysis of the literature. This paper utilizes articles from existing literature that explores the complexities and challenges facing mental health businesses in the pandemic. The value of a content analysis review of the literature is the ability to take dispersed, innovative perspectives and emerging research on a topic and articulately merge them for practitioner digestion or as a base-level foundation for future academic research. Databases used include ResearchGate, Academia.edu, Google Scholar, Business Source Corporate, ProQuest Business, and ProQuest News. Keywords included COVID-19 and counseling, Business Strategy and COVID-19, Change Management, Management Strategy, Strategic Planning, Planned Change, telemedicine, and Tele-counseling. The focus combined the use of seminal literature and current literature in 5 years.

Organizational development action research is a methodical examination approach to analyze real-world problems, establish evidence, apply established theories, engage in data collection interventions, present reliable results, and develop viable solutions (Stringer \& Aragón, 2020; Masters, 1995; O’Brien, 1998).

This approach is the core methodology for management and organizational development consultants (Stringer \& Aragón, 2020; Cheung-Judge and Holbeche, 2015). The ability to marry practical and theoretical frameworks in the study of complex organizations in real-time highlights the worth, value, and importance of organizational development action research as an approach (Stringer \& Aragón, 2020; Masters, 1995; O’Brien, 1998).

\section{Strategic Management Process Framework}

Developing a strategic market management process takes intentionality and commitment. However, when an organization designs its strategy, develops an infrastructure to sustain it, and implements the procedure, it will be poised to reap the process's benefits. After much review, the management consultant chose to use Aaker's strategic market management process framework to design and manage the strategic changes needed to improve business. The management consultant believes Aaker's framework is a straightforward structure and is conducive to integrating other organizational and change models into the framework as needed. Aaker's framework for strategic market management has two main components: (1) strategic analysis and (2) strategic identification and selection (2001). 


\section{Strategic Analysis}

The strategic analysis focuses on (a) external analysis and (b) internal analysis. The internal analysis examines what is happening inside the organization. Using Aaker's framework, organizations would focus on performance analysis and determinants of strategic options. Performance analysis examines an organization's financial performance and non-financial performance indicators, such as customer satisfaction/brand loyalty, product/service quality, and even manager/employee capability and performance (Aaker, 2001).

In contrast, the external analysis examines outside factors that impact an organization. Organizations should examine their customers' unmet needs and customer segments; competitors' objectives, cost strategy, and culture; the market's profitability and growth projections; and external forces such as technological advancements, governmental and economic policies, and even societal/ cultural shifts (Aaker, 2001).

\section{Strategic Identification and Selection}

The strategic analysis results help generate strategic alternatives and provide criteria to select from among the options (Aaker, 2001). Strategic identification and selection include (a) identifying the strategic opportunities, (b) selecting a strategy, (3) implementing the operating plan, and (d) reviewing the strategies (Aaker, 2001).

\section{Organizational Diagnosis Using SWOT Method}

Using the findings from the strategic analysis (external and internal) requires organizational leaders to decide how to distill and organize these data points. Categorizing and clarifying data from the strategic analysis brings value to the data and prepares it to be actionable and not merely informative. Moreover, it positions the organization's leaders to make an accurate diagnosis of the organization.

To categorize the data point from the strategic analysis, the management consultant used the SWOT analysis method given its ease of incorporation into Aaker's framework. Aaker notes that the outcomes of the strategic analysis include opportunities, threats, trends, and strategic uncertainties (these emerge following the external analysis); and strategic strengths, weaknesses, problems, constraints, and uncertainties (these emerge from the internal analysis) (Aaker, 2001). The management consultant noted that these outcomes aligned with the SWOT Method elements, making it prudent to use the SWOT method to analyze the data.

A SWOT analysis used by the management consultant allows data to be distilled into the four quadrants, including strengths, weaknesses, opportunities, and threats. Subsequently, organizations can use this to diagnose the organization. Organizational diagnosis is a critical step for any change initiative. Noolan (2006) notes that organization diagnosis can identify that which "is" and that which "could be" and that a diagnosis serves to eliminate the gap between the two. Table 1 shows the SWOT findings and the diagnosis of TCL:

\section{Diagnosis}

TCL has several strengths and opportunities that make the practice conducive to undergo a robust change initiative, to include (1) staff have already begun discussions to move to a tele-counseling model, (2) the clinicians have a robust social media presence that could assist with their transition to a tele-counseling or telemedicine model. Telemedicine or tele-counseling is the use of a medical field strategy focused on automation and technology to treat underserved and remote patients (Ivy, 2018). In practice, telemedicine encompasses a therapist in one location treating a patient in a different location, like the patient's home, through video conferencing tools (Ivy, 2018). The technology used to enable this remote medical appointment is usually video conference software, which leverages the internet for communications (Ivy, 2018). 
Table 1. SWOT findings and the diagnosis of TCL

\begin{tabular}{|c|c|}
\hline Strength & Weakness \\
\hline $\begin{array}{l}\text { - Each clinician is paid using a commission-based model. } \\
\text { - Each clinician, except for the CEO, receives the same } \\
\text { base salary as part of their compensation model. • The } \\
\text { office manager is paid on an hourly wage. } \\
\text { - There has been positive discussion before COVID about } \\
\text { transitioning to tele-counseling to reduce their physical } \\
\text { footprint and reduce office space costs. } \\
\text { - The CEO and clinicians are deeply connected to their } \\
\text { mission to provide high-quality, continuous care. } \\
\text { - The practice currently has minimal overhead costs due to } \\
\text { its lean operating structure. } \\
\text { - Conducts a quarterly customer satisfaction and needs } \\
\text { assessment (from both a clinical and customer service- } \\
\text { based lens). Thus, regularly has a current pulse on client } \\
\text { needs. } \\
\text { - CEO willing to have clinicians to become partners } \\
\text { - All clinicians have extensive face to face counseling } \\
\text { experience. None have ever used tele-counseling. }\end{array}$ & $\begin{array}{l}\text { - TCL has five full-time clinicians on staff. Each work } \\
\text { from the office spaces provided by the owner, The } \\
\text { management consultant. } \\
\text { - The CEO receives a higher base salary, which } \\
\text { compensates her for additional business development } \\
\text { services she undertakes beyond the client caseload. } \\
\text { - This is the first change initiative the CEO has ever } \\
\text { undertaken. } \\
\text { - The practice is not currently designed to deliver tele- } \\
\text { counseling. } \\
\text { - The Office Manager manages payroll and provides } \\
\text { administrative support. } \\
\text { - The practice's most considerable expense outside of } \\
\text { payroll is building space. } \\
\text { - The only revenue stream is payment for clinical services, } \\
\text { and this is on a decline. } \\
\text { - A sharp decline in payments due to a reduction in } \\
\text { services provided } \\
\text { - The business brand is not widely known. Most marketing } \\
\text { is word of mouth or done by the CEO } \\
\text { - Clinicians are concerned about losing jobs due to a sharp } \\
\text { decline in billable hours for patients. }\end{array}$ \\
\hline Opportunity & Threat \\
\hline $\begin{array}{l}\text { - Each clinician and the office manager have a robust } \\
\text { social media presence that connects to mental health. } \\
\text { - COVID gives a chance to reach more people from one } \\
\text { location and the opportunity to offer other services } \\
\text { - Expand digital footprint to expand the client base } \\
\text { - Opportunity to diversify services that can be delivered } \\
\text { remotely even beyond COVID } \\
\text { - Tele-counseling is a growing provider method and } \\
\text { becoming more widely accepted, which COVID may } \\
\text { accelerate this. } \\
\text { - Opportunity to expand existing customer profiles and } \\
\text { create new profiles for new client segments }\end{array}$ & $\begin{array}{l}\text { - Patients may lack access to reliable internet to receive } \\
\text { constant services. } \\
\text { - Privacy concerns surrounding telehealth services. } \\
\text { - Will the patient's insurance company cover this method } \\
\text { of providing services? } \\
\text { - State boundaries may limit or dictate to an extent how } \\
\text { the practice can provide tele-counseling } \\
\text { - HIPPA requirements may limit what can be done }\end{array}$ \\
\hline
\end{tabular}

\section{Review of the Literature}

According to the American Counseling Association (ACA), tele-counseling is "the provision of counseling services by means other than face-to-face meetings, usually with the aid of technology" (ACA, 2014, p. 20). The practice of distance counseling requires an understanding of the unique characteristics of counseling via technology. It requires organizations to change their organizational structure, employee training, leadership approaches, and client engagement approaches (DuPont, 2019). Organizations that introduce these new technologies as a part of their business strategy require resources dedicated to training, hardware, and software (DuPont, 2019). Technological tools such as computers with webcams and the accompanying software need to be used effectively, especially regarding the competent delivery of therapy services (DuPont, 2019).

Glueckauf et al. (2018) research investigated the use of automation and technology in mental health practice. Glueckauf et al. (2018) surveyed mental health clinicians in the U.S. concerning their attitudes about what automation and communication technologies are most valuable with telecounseling. The research results found that $72.56 \%$ of the respondents considered video-conferencing useful for distance counseling (Glueckauf et al., 2018). 
Tele-counseling, both patients and clinicians comprehend the unique characteristics of counseling appointments that occur virtually or remotely (Glueckauf et al., 2018; Dupont, 2019). Distance counseling methods utilize a greater reliance on the written and oral communication between counselor and client because there is a lack of visual cues and nonverbal communication present in face-to-face counseling (Glueckauf et al., 2018, Dupont, 2019). Videoconferencing tools can enhance virtual counseling's optical characteristics, but the technology itself can be complicated for both client and counselor (Glueckauf et al., 2018, Dupont, 2019). The introduction of telemedicine as a business strategy requires an understanding of the problematic aspects of change management.

\section{Change Management}

Change, large or small, can have varying degrees of effects that can ripple throughout an organization. Most organizations experience non-invasive, continuous change, also known as evolutionary change (Burke, 2018). However, a less common type of change is a revolutionary change. Revolutionary change is highly invasive, as it often changes the organization's deep structure (Burke, 2018). Whether organizations undergo revolutionary or evolutionary change, organizational leaders must consider the impact of change on staff and the organization.

According to Burke (2018), change can happen at three (3) levels in an organization, including the individual, group, and system levels. Leaders should consider the possible responses to change at each level. For example, employees may resist the change being proposed at the individual level because it brings feelings of loss (Burke, 2018). At the group level, Burke (2018) notes that the more work units become actively engaged in helping plan and implement the change, the company is more likely to embrace the change. At the systems level, change is highly strategic. The critical objective for system-level change is to solidify the scope of change desired and the appropriate change model to employ. Given the organizational diagnosis of TCL, it is possible that TCL would benefit from incorporating a corporate design model to help design the change and then an appropriate change model to execute the design.

Ultimately, as leaders prepare their organizations for change, it must anticipate roadblocks to change and employee responses to change, design the change initiative, and implement the change.

\section{Leadership Style}

At the heart of successful change initiatives are strong leadership. In an environment where uncertainties are high and change occurs frequently, strategic, adaptive, transformational, and technical leadership is essential.

\section{Strategic Leadership}

Organizational leaders often grapple with deciding the entrepreneurial and managerial strategy to employ when navigating their specific market. Simon Sinek recommends using game theory when approaching strategic leadership. Sinek (2016) suggests that leaders see business as an infinite rather than finite game. Playing an infinite game requires organizational leaders to be singularly focused on their specific, core mission.

To curb the revenue decline during the COVID-19 pandemic, organizations may be tempted to play a finite game that leads them to be more shortsighted, allowing COVID to change the "why" of their business haphazardly and inauthentically. Thus, organizations must decide to hone their mission or expand it because it will be a wise decision post-COVID. According to Sinek (2016), great leaders are aware of the infinite game and intentionally play it.

\section{Transformational Leadership}

In addition to strategic leadership, adopting a transformational leadership style during and postCOVID can benefit organizations, no matter the industry because transformational leaders can create a positive work culture for their employees (DiFranza, 2019). At its core, transformational leadership 
inspires employees to look ahead, focusing on the greater good and with an expressed intention to move as a single unit with a common goal (i.e., mission) (DiFranza). Still, in the era of COVID, leaders must be able to discern between even more nuanced styles of leadership, such as adaptive and technical leadership.

\section{Adaptive and Technical Leadership}

Technical problems call for technical leadership, and adaptive challenges warrant adaptive leadership (Heifetz et al., 2003). Technical issues, which tend to be more concrete, benefit from leadership that has answers, is decisive and can map the future as in the landing point, and overall can make difficult problems simple (Heifetz et al., 2003). In contrast, adaptive challenges are more abstract, less straightforward, and require the organization to learn new ways (Heifetz et al., 2003). Thus, adaptive leadership is usually best since adaptive leaders can move the organization to face the problems for which there are no simple, painless solutions and push the organization to a learning focus (Heifetz et al., 2003).

Leaders that can navigate fluidly between technical and adaptive leadership are ideal change leaders. This ability is akin to being able to adopt a learning and performance orientation. Goal orientation theory, which comprises the two orientations, are defined as follows:

Learning (Mastery) Goal Orientation: Learning oriented persons are interested in increasing their competence; it is "a purpose of personal development and growth that guides achievement-related behavior and task-engagement" (Kaplan \& Maehr, 2007).

Performance Goal Orientation: Performance-oriented persons are interested in demonstrating their competence. However, studies show that performance-oriented goals foster avoidance of challenging tasks due to anxiety about failure (Dweck \& Leggett, 1988).

If leaders avoid making fear-based decisions, then navigating an orientation's culture fluidly would be essential, mostly if this leadership approach is adopted at all leadership levels in the organization.

\section{Organizational Resilience}

COVID has proven to be quite disruptive to almost every industry. Thus, organizations have had to become adaptable, flexible, and have a learning and creative builder mentality. To accomplish this, David Loree (2020) recommends that organizations strive to be resilient organizations, which Loree considers an organizational coping response to industry stress and threats. Loree (2020) describes resilient organizations as facing reality, searching for meaning, and having ritualized ingenuity. In the current pandemic, these attributes are essential. Watson (2020) admonishes leaders to avoid being dishonest with employees about the pandemic and the subsequent organizational state.

While it may seem obvious for leaders to be honest, especially in a pandemic, if leaders lack personal resilience in crisis and the organization is not resilient by design and within its culture, leaders may lean more on their coping skills during stress, and that can manifest as not facing reality, ergo, being dishonest. This brings to the surface a poignant discussion about leader resilience. While organizations may want to be resilient, leaders will play a key role in creating such an organization. If leaders cannot overcome feelings of organizational nostalgia, as noted by Batat (2020), and feelings of fear and denial, then their inability to cope may transfer to their staff.

One way for leaders to become resilient and build resilient organizations is to have an explanatory style that shapes a resilience culture. In the context of organizational leaders, an illustrative style is how a leader habitually chooses to explain and categorize various events that can directly impact their organization's overall health and trajectory (Abramson et al., 1978). One's explanatory style is essential because it can predict a person's behavior in crises, especially in decision-making (Satterfield, 1998). Specifically, a leader's descriptive style can drive their decision to persevere or acquiesce in the face of adversity (Hansen, 2016).

To further underscore resilience and its connection to an explanatory style, one's descriptive style can interact with a person's existing heuristic models. Heuristics are cognitive shortcuts, often 
attributed to poor decision-making (Satterfield, 1998; Stanovich, 2018). Satterfield's (1998) found that "optimism, low integrative complexity, and the interaction between the two significantly predicted high levels of aggression and risk-taking behaviors. Conversely, pessimism, high integrative complexity, and their interaction were predictive of subsequent passivity and caution."

Optimism and pessimism describe one's explanatory style. At the same time, integrative complexity is the degree to which thinking, and reasoning involve the recognition and integration of multiple perspectives and possibilities and their interrelated contingencies, which links to heuristics (Békés et al., 2019). These findings reveal that a person with an optimistic explanatory style (i.e., Explains events as temporary, local, and changeable [Hansen, 2016]) but engages in heuristics reflective of low integrative complexity may make impulsive decisions that are short sided.

\section{Continuity of Care (CoC)}

$\mathrm{CoC}$ is concerned with the quality of care over time (Gulliford et al., 2006). Research has shown that $\mathrm{CoC}$ is positively correlated with service satisfaction and quality of life (QoL) (Loranger et al., 2020). However, to best understand how to deliver $\mathrm{CoC}$, mental health providers should examine CoC's variable through the lens of care recipients.

In response to COVID, owners of mental health practices must determine how to ensure CoC for their care recipients. Specific considerations for continuity of care, including how to move to a tele-counseling model, identifying COVID-specific tele-counseling resources, identifying insurance coverage challenges for patients receive care via tele-counseling, and being aware of HIPPA and state requirements.

\section{The success of the Tele-Counseling Model}

Tele-counseling is any psychological service performed over the internet, including emails, chat rooms, and web cameras (De Luca \& Calabrò, 2020). Tele-counseling ranges from an individual, couple, and group psychotherapy, provided by qualified, credentialed therapist (De Luca \& Calabrò, 2020). Since the onset of COVID, tele-counseling is an effective treatment delivery method (De Luca \& Calabrò, 2020). Ultimately, tele-counseling can help address COVID-19 and other pandemic situations by limiting exposure to infection for vulnerable populations and health care workers (TRC, 2020). Also, tele-counseling can expand the reach of resources to communities that have limited access to needed services. This allows patients to receive health services away from infectious populations, such as hospitals, health clinic waiting rooms, private practices (TRC, 2020).

\section{State Guidelines for Tele-Counseling}

Every state has specific guidelines and boundaries for tele-counseling. Firms looking to move to this service delivery model must research their state laws and policies governing tele-counseling and make sure their business aligns with these policies (Hill, 2020). When firms fail to comply with state guidelines, they risk losing their business and health licensure needed to legally provide services (Hill, 2020) and thus, disrupt CoC.

\section{Insurance Reimbursement}

Research suggests that more and more insurance providers cover online therapy sessions and thus agree to reimburse the cost of care (Gillihan, 2020). To help ensure CoC, TCL must assess how moving to a tele-counseling model will impact patients' insurance coverage.

\section{Tele-Counseling Technical Platform}

At the heart of tele-counseling is technology. Organizations must select a platform that supports the firm's needs and the patient's needs. To date, there are a variety of platforms available, such as Zoom, Microsoft Teams, WhatsApp, and more (Hill, 2020). Typically, before selecting a platform, the organization would need to confer with HIPPA guidelines to see if the platform is HIPPA- 
compliant (Hill, 2020). However, HIPPA has relaxed some of its policies given COVID (Hill, 2020). Thus, organizations moving to this model must decide how best to proceed and consider the risks and benefits (long-term and short-term) to choosing a platform that may be compliant in a COVID environment but not in a post-Covid climate.

Achieving $\mathrm{CoC}$ from a technical standpoint is critical. However, there are strategic considerations that play into $\mathrm{CoC}$, such as market expansion.

\section{Strategic Market Expansion}

While playing the infinite game is critical to a long-term strategy, as noted by Sinek (2016), organizations should recognize that COVID presents a unique opportunity for organizational leaders to re-examine their core mission and core approach to accomplishing their mission. Many state and local governments are aiding firms during COVID. Thus, a strategic change or expansion in mission can be advantageous for organizations, tiny businesses, such as TCL. Ultimately, organizations must evaluate their sustainable competitive advantage (SCA).

An organization's sustainable competitive advantage (SCA) is its ability to achieve and maintain strategic assets and strategic competencies that distinguish the organization from its competitors (Aaker, 2001). When viewing an organization's SCA through the strategic management process, organizations can explore opportunities to create strategic opportunities to promote their strategic positioning and advantage.

One aspect to consider when looking to create an SCA is the role of microeconomics. Microeconomics studies individual consumers and firms' decisions and examines prices and production in single markets and the interaction between different needs (Chappelow \& Barnier, 2020). Understanding and being able to predict consumer trends can inform an organization's ability to create an SCA. Consider the utility theory in microeconomics. This theory states that consumers purchase and consume goods that will maximize their happiness or "utility" within the confines of their available income (Chappelow \& Barnier, 2020). For example, when organizations consider economic, demographic information, such as the mean income of residents in a certain radius of its brick and mortar location, one might assess the likelihood of a person to drive X-miles and to spend X-dollars each month or on certain items. The COVID environment warrants a utility review to determine what will make patients happy in this new environment and a post-Covid climate. With this information, organizations can predict and plan for new service and product rollouts, and more.

Lastly, another aspect to consider when examining how to develop an SCA is to consider how organizations will connect with existing and potential customers in a COVID environment (i.e., how will an organization market in a COVID era)? This is important given that Hill states that marketing in tele-counseling can be challenging (Hill, 2020). In summary, the literature suggests that TCL should focus on its leadership approach to change management, determining ways to ensure $\mathrm{CoC}$, and explore ways to expand their reach in the market.

\section{Consultant Recommendations}

Before crafting a staff recommendation, the management consultant had to determine if TCL would need to undergo a revolutionary or evolutionary change. Based on the diagnosis and a review of the literature, the management consultant decided that, ultimately, the practice had to undergo both a revolutionary and evolutionary change in how services are delivered, including the use of technology to engage clients virtually.

TCL plans to expand its mission to commit to education and awareness of mental health from a revolutionary lens. This new scope will create opportunities to leverage untapped competencies within the staff and elevate them to organization competencies. This will help them improve their SCA. From an evolutionary lens, the practice must make several processes and system changes to change how they currently administer their existing mission to provide high-quality, continuous care. 
After reviewing relevant literature, the management consultant has crafted a set of strategic recommendations for TCL staff and clients. Each recommendation is the product of the management consultant's review of research literature and reputable articles about counseling, telehealth, change management, and more. The management consultant's recommendations are to:

- Select an organizational design model and change model to design the future state of TCL. The management consultant recommends using the STAR model to re-design TCL and the Burke-Litwin model to plan the change initiative. Using the STAR model, the management consultant must define desired organizational performance outcomes during COVID and postCOVID and the STAR components, which include strategy, people, processes, rewards, and structure (Galbraith, 2002). The management consultant will use this design model's outputs as the inputs for the Burke-Litwin change model.

- Plan the communications plan to support TCL's pivot. Based on the leadership literature, change management literature, and continuity of care literature, the management consultant realizes that planning how to communicate change is essential to helping staff buy-in to change and reduce the sense of loss that change can often bring. Moreover, planning how to communicate change can create a sense of safety for patients, especially during a time of great uncertainty. Moreover, from an SCA perspective, planning how to share this change with the market will impact TCL's successful transition to tele-counseling.

- Select a tele-counseling platform and make telemedicine a key business strategy. While it may seem prudent to pick a platform that will be HIPPA-compliant today and tomorrow, the management consultant recognizes that not all patients have access to HIPPA-compliant technology like Zoom. Thus, to sustain $\mathrm{CoC}$, the management consultant recommends offering all available platforms that HIPPA allows during COVID to increase care access. Moreover, the management consultant plans to recommend staff work with patients to gradually connect to resources that can help them secure access to more compliant platforms or slowly transitioning them to other practices that will revert to in-person therapy post-COVID.

- Improve SCA. The management consultant recommends that TCL build an education and awareness component as a new business line. With COVID, clinicians can book TCL to do virtual seminars to become new revenue for the practice. Using the clinician's existing social media reach, TCL can acquire new customers and enter market segments they originally were not in. The management consultant also recommends that the practice consider interviews with local news and media outlets to share how uniquely qualified TCL is to provide online therapy.

- Capture and maintain a Telehealth resource log. Several organizations developed resources that can help set up telehealth programs to address COVID-19. The management consultant recommends cataloging this information, so it is an available resource for the practice. This catalog will include a network of local counselors, social workers, doctors, etc., research literature, videos, toolkits, and more.

- Adopt new leadership styles. Lastly, the management consultant recognizes to ensure the health and longevity of the practice. The CEO must adjust her leadership style. The management consultant believes incorporating transformational and adaptive leadership attributes would be beneficial to better-supporting staff and helping to lead this change initiative.

\section{RECOMMENDATIONS FOR FUTURE RESEARCH}

There could be tremendous value in a qualitative study that engages patients to find out about their lived experiences using virtual counseling. This approach would explore the kind of support, training, and their feelings about advantages and challenges to using remote counseling. 


\section{REFERENCES}

Aaker, D. A. (2001). Developing business strategies (6th ed.). John Wiley \& Sons, Inc.

Abramson, L. Y., Seligman, M. E. P., \& Teasdale, J. D. (1978). Learned helplessness in humans: Critique and reformulation. Journal of Abnormal Psychology, 87(1), 49-74. doi:10.1037/0021-843X.87.1.49 PMID:649856

American Counseling Association. (2014). Code of ethics. Retrieved from https://www.counseling.org/resources/ aca-code-of-ethics.pdf

Batat, W. (2020). The Business of Recovery. https://iveybusinessjournal.com/the-business-of-recovery/

Békés. (2019). Vera \& Suedfeld, Peter. Integrative Complexity. doi:10.1007/978-3-319-28099-8_1942-1

Burke, W. W. (2018). Organization Change: Theory and Practice (5th ed.). Sage.

Chappelow, J., \& Barnier, B. (2020). Microeconomics. Investopedia. Retrieved from: https://www.investopedia. com/terms/m/microeconomics.asp

Cheung-Judge, M.-Y., \& Holbeche, L. (2015). Organization Development: A practitioner's guide for O.D. and H.R. Kogan Page.

De Luca, R., \& Calabrò, R. S. (2020). How the COVID-19 Pandemic is Changing Mental Health Disease Management: The Growing Need of Telecounseling in Italy. Innovations in Clinical Neuroscience, 17(4-6), 16-17. PMID:32802587

DiFranza, A. (2019). Transformational leadership: How to inspire innovation in the workplace. Northeastern University Graduate Programs. https:/www.northeastern.edu/graduate/blog/transformational-leadership/

DuPont, C. (2019). The relationship between counselor educator technology self-efficacy and distance counseling skills education (Order No. 27547548). Available from ProQuest Dissertations \& Theses Global.

Dweck, C. S., \& Leggett, E. L. (1988). A Social Cognitive Approach to Motivation and Personality. Psychological Review, 95(2), 256-273. doi:10.1037/0033-295X.95.2.256

Emerald, G. (Ed.). (2005). Strategic planning. ProQuest Ebook Central: https://ebookcentral.proquest.com

Gillihan, S. (2020). How to transition to seeing your therapist online. Psychology Today.

Gulliford, M., Naithani, S., \& Morgan, M. (2006, October). What is 'continuity of care'? Journal of Health Services Research \& Policy, 11(4), 248-250. doi:10.1258/135581906778476490 PMID:17018200

Glueckauf, R. L., Maheu, M. M., Drude, K. P., Wells, B. A., Wang, Y., Gustafson, D. J., \& Nelson, E.-L. (2018). Survey of psychologists' telebehavioral health practices: Technology use, ethical issues, and training needs. Professional Psychology, Research and Practice, 49(3), 205-219. doi:10.1037/pro0000188

Hansen, N. (2016, August 4). Strategy as Choice [Video]. YouTube. https://www.youtube.com/ watch?v=lQwHlptnYqk

Heifetz, R. A., \& Laurie, D. L. (2003). The Leader as Teacher: Creating the Learning Organization. Ivey Business Journal.

Hill, K. (2020). How to move your practice entirely online. Brighter Vision. https://www.brightervision.com/ coronavirus-prep-your-practice-1/

Ivy, O. (2018). Measuring patient trust in their physician and its impact on telemedicine (Order No. 10842600). Available from ProQuest Dissertations \& Theses Global.

Kaplan, A., \& Mahr, M. L. (2007). The Contributions and Prospects of Goal Orientation Theory. Educational Psychology Review, 19(2), 141-184. doi:10.1007/s10648-006-9012-5

Krogerus, M., \& Tschappeler, R. (2018). Decision Book: Fifty Models for Strategic Thinking. Norton \& Company, Incorporated, W.W.

Loree, D. (2020). Building Corporate Resilience. Ivey Business Journal. https://iveybusinessjournal.com/ building-corporate-resilience/ 
Masters, J. (1995). The history of action research. Action Research Electronic Reader.

Noolan, J. (2006). Organization diagnosis phase. In B.R. Jones \& M, Brazzel (Eds.), The NTL handbook of organization development and change (pp. 192-221). San Francisco, CA: Wiley.

O'Brien, R. (1998). An overview of the methodological approach of action research. Academic Press.

TRC. (2020). Telehealth resources to address COVID-19. National Consortium of Tele-health Resources Centers (TRC). https://www.telehealthresourcecenter.org/covid-19-resources/

Satterfield, J. M. (1998). Cognitive-affective states predict military and political aggression and risk-taking: A content analysis of Churchill, Hitler, Roosevelt, and Stalin. The Journal of Conflict Resolution, 42(6), 667-690. doi:10.1177/0022002798042006001

Sinek, S. (2016, Nov 2). Most Leaders Don't Even Know the Game They're In [Video]. YouTube. https://youtu. be/RyTQ5-SQYTo

Stanovich, K. E. (2018). Miserliness in human cognition: The interaction of detection, override, and mindware. Thinking \& Reasoning, 24(4), 423-444. doi:10.1080/13546783.2018.1459314

Stringer, E. T., \& Aragón, A. O. (2020). Action research. Sage Publications.

Watson, T. (2020). How to Govern, Manage, and Work Amid COVID-19. https://iveybusinessjournal.com/howto-govern-manage-and-work-amid-covid-19/

De Andra Judge holds a B.S. in Psychology from Howard University and a M.A. in Industrial and Organizational Psychology from The Chicago School of Professional Psychology (TCSPP). Judge is earning a PHD in Business Psychology, with a concentration in Consulting.

Darrell Norman Burrell is post graduate student and a 2017 graduate of the National Coalition Building Institute's (NCBI) Leadership Diversity Institute. He is a Certified Diversity Professional. He is an alumnus of the prestigious Presidential Management Fellows Program www.pmf.gov. Dr Burrell has a doctorate degree with majors in Education and Executive Leadership Coaching from A.T. Still University. Dr. Burrell has an Education Specialist (EdS) graduate degree in Higher Education Administration from The George Washington University. He has two graduate degrees one in Human Resources Management/Development and another Organizational Management from National Louis University. He also has a Master of Arts degree in Sales and Marketing Management from Prescott College. He has extensive years of university teaching experience at several universities. 